\title{
The state of vascular access teams: results of a European survey
}

\author{
Noemí Cortés Rey ${ }^{1}$, Fulvio Pinelli ${ }^{2}$, Fredericus van Loon ${ }^{3}$, Jennifer Caguioa ${ }^{4}$, Gema Munoz \\ Mozas $^{5}$, Vincent Piriou ${ }^{6}$, Ulf Teichgräber ${ }^{7}$, Didier Lepelletier ${ }^{8}$, and Baudolino Mussa ${ }^{9}$ \\ ${ }^{1}$ Hospital Teresa Herrera, Complejo Hospitalario Universitario de A Coruña \\ ${ }^{2}$ University Hospital Careggi \\ ${ }^{3}$ Catharina Hospital \\ ${ }^{4}$ King's College Hospital NHS Foundation Trust \\ ${ }^{5}$ Royal Marsden NHS Trust \\ ${ }^{6}$ Hospices Civils de Lyon \\ ${ }^{7}$ University Hospital Jena \\ ${ }^{8}$ University Hospital Centre Nantes \\ ${ }^{9}$ University of Turin
}

July 1, 2021

\begin{abstract}
Background: Many European health institutions have appointed multidisciplinary teams for the general management of vascular access to help improve efficiency, patient safety and reduce costs. Vascular access teams (VATs), or infusion teams, are specifically trained groups of healthcare professionals who assess, place, manage and monitor various outcomes and aspects of vascular access care. Objective: To assess the current landscape of vascular access management as a discipline across Europe. Methods: A Faculty of European VAT leads and experts developed a survey of 20 questions which was disseminated across several European countries. Questions focused on respondent and institution profile, vascular access device selection and placement, monitoring and reporting of complications, and access to training and education. The 1449 respondents included physicians, nurses, anaesthetists, radiologists and surgeons from public and private institutions ranging in size. Results: Availability of dedicated VATs vary by country, institution size, and institution type. Institutions with a VAT are more likely to utilise a tool (e.g., algorithm or guideline) to determine the appropriate vascular access device $(55 \%$ vs $38 \%$, p < 0.0002$)$ and to have feedback on systematic monitoring of complications $(40 \%$ vs $28 \%, \mathrm{p}=0.015)$. Respondents from institutions with a VAT are more likely to have received training on vascular access management $(79 \%$ vs $53 \%, \mathrm{p}<0.0001)$ and indicated that the VAT was a source of support when difficulties arise. Conclusion: The survey results highlight some of the potential benefits of implementing a dedicated VAT including use of a broader range of vascular access devices, increased awareness of the presence of vascular access policies, increased likelihood of recent vascular access training, and increased rates of systematic monitoring of associated complications. The study reveals potential areas for further focus in the field of vascular access care, specifically examining the direct impact of vascular access teams.
\end{abstract}

\section{Hosted file}

VAT survey manuscript_submission_main text.docx available at https://authorea.com/users/ 422980/articles/528568-the-state-of-vascular-access-teams-results-of-a-european-survey 
A Is there a dedicated vascular access team/service in your institution?

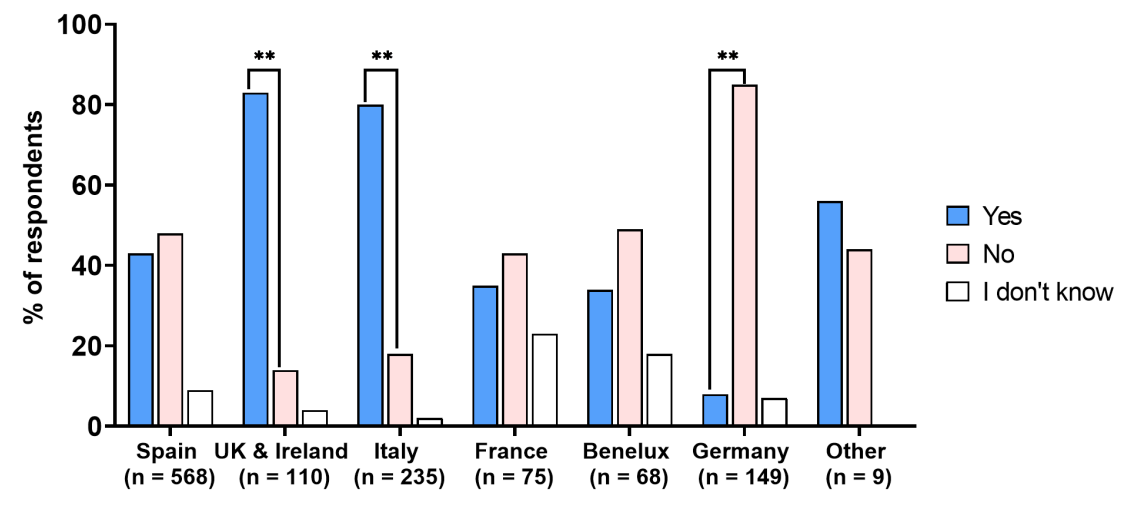

B Which best describes your institution size?

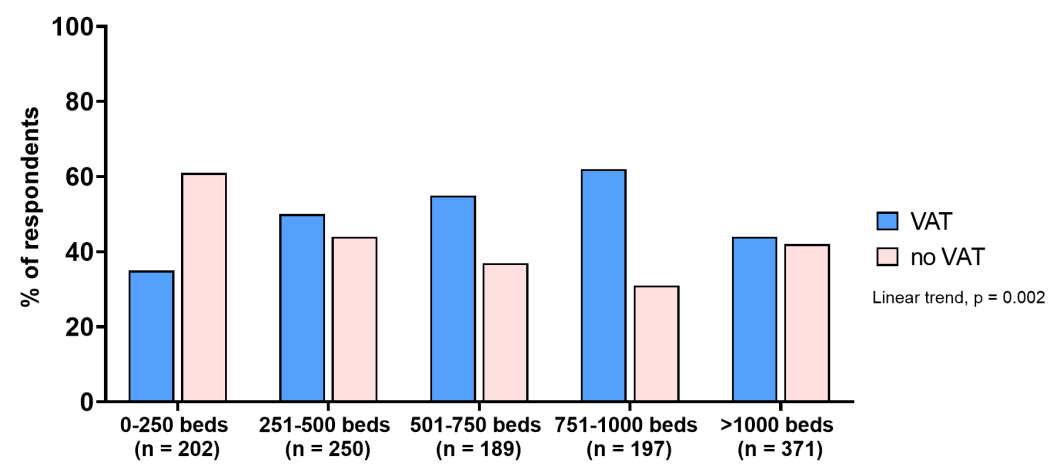

C Which best describes your institution type?

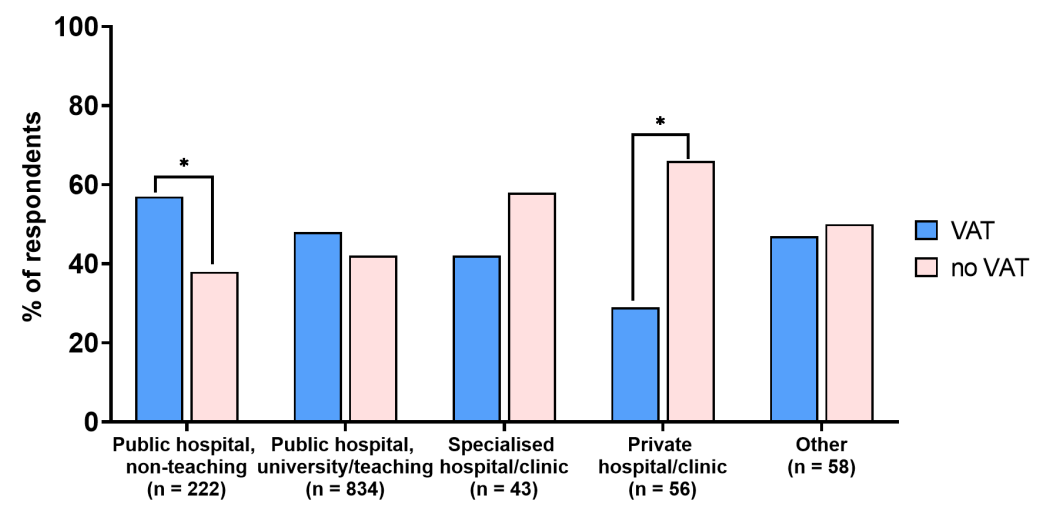


A Do you use a tool in your institution to help define the type of vascular access device that is most suitable for the patient and its IV therapy?

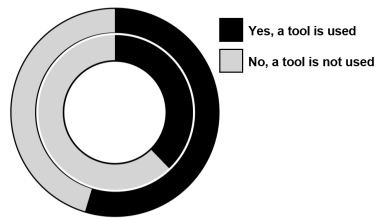

B Which of the following is the most appropriate description of the tool?

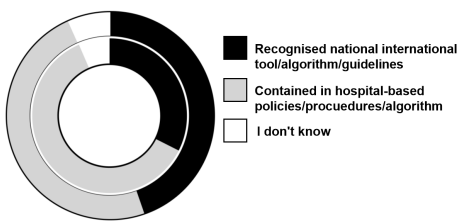

C In your role, which of the following types of vascular access devices do you place?
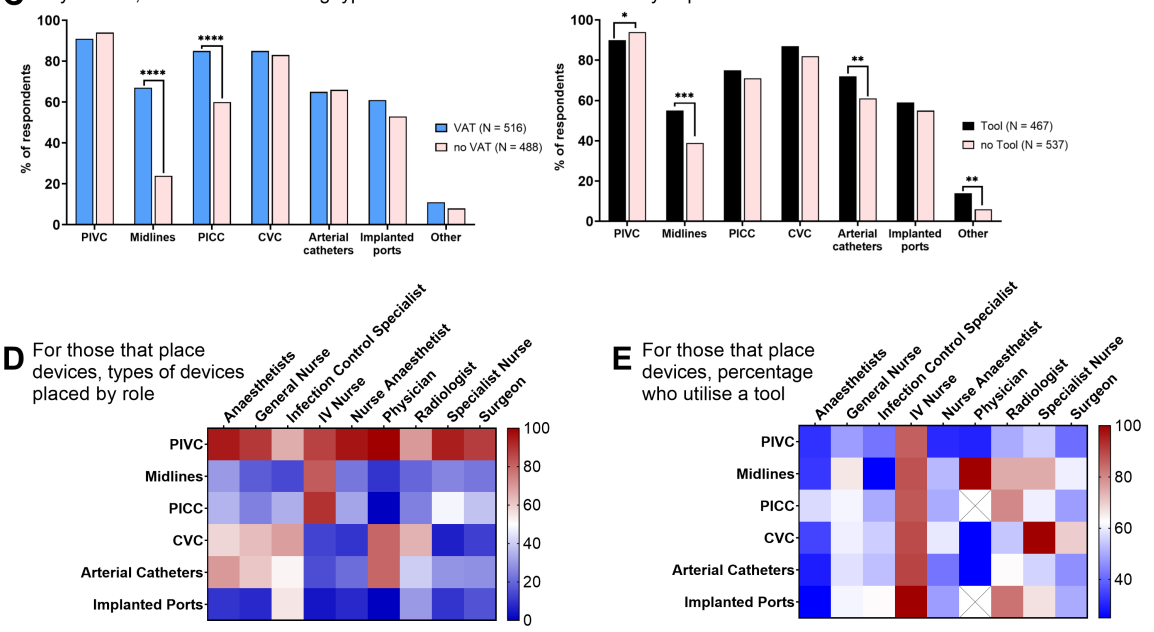

A Which of the following complications are systematically monitored and reported in your hospital information system for central devices and for peripheral devices?

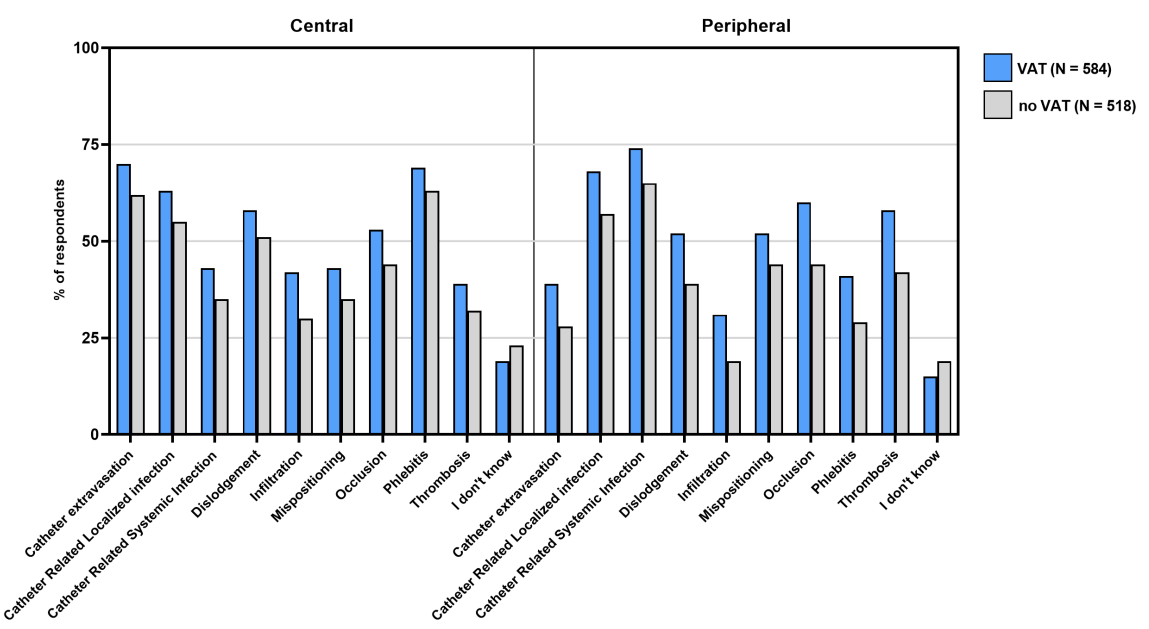

B Do you get feedback on the complication monitoring data from your hospital

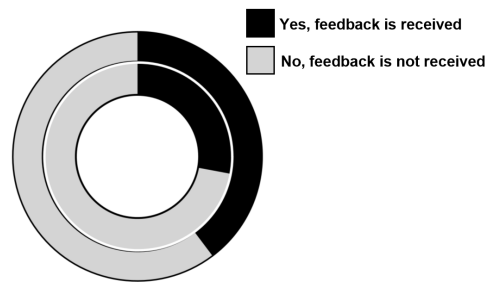


A Have you received a vascular access

B Where did you receive your training

C In which of the following topics have you

the last 3 years? ?

received a vascular access training/update?
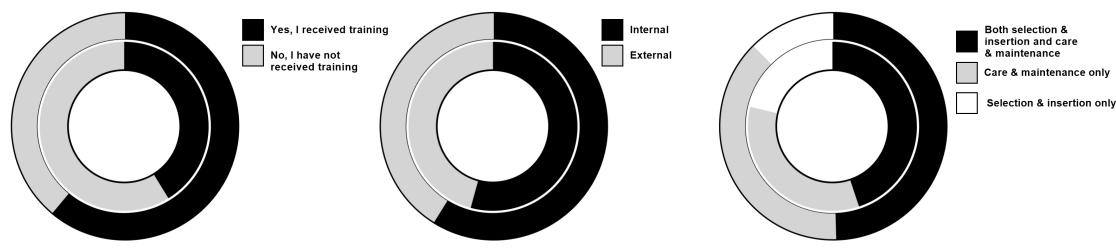

A Who do you contact when you have difficulty

B Do you know if your hospital has vascular

C What are your most important in placing a vascular access?

B access or IV therapy policies?
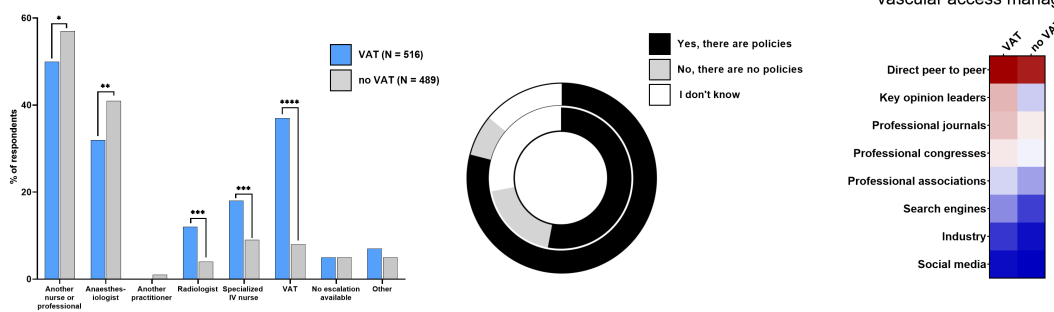